\title{
The Absolute Radiometric Calibration Facility ARCF 2.0 at TNO
}

\author{
B. Gür, G. Otter, R. Jansen, J. Groote-Schaarsberg, S. Brinkers \\ TNO Science and Industry, Stieltjesweg 1, 2628CK Delft, The Netherlands
}

\begin{abstract}
Optical components with scattering properties, so-called diffusers, are core elements of calibration units in earth observation instruments. Their performance influences significantly the achievable accuracy of scientific observations. It is of high importance however to characterize the scattering properties of such a diffuser with minimum uncertainty onground before launched into orbit and before being utilized in its calibration purpose. Over the past decades TNO has operated an "Absolute Radiometric Calibration Facility (ARCF)" to ensure such accurate characterization of space components. In the recent past TNO has put increased efforts in upgrading and modernizing its facility into a modern high class facility (ARCF 2.0) to measure scattering properties of a variety of materials and components to meet the growing demands for accurate measurements for space applications. This paper describes the above mentioned facility ARCF 2.0 with its unique measurement capabilities and outlies several examples.
\end{abstract}

Keywords: BSDF, Diffuser, Radiometric Calibration

\section{INTRODUCTION}

Earth observation instruments have emerged rapidly in the past decades and have greatly improved our understanding of our planet. Significant progress in various technologies has allowed scientific observations from space with a high level of accuracy. Accuracy demands will remain or be even more stringent for future missions. On-board calibration units are highly important elements of an earth observation instrument, ensuring through its calibrating and monitoring functionality accurate performances and thus accurate scientific observations of an instrument during its lifetime in orbit. For example, instruments for spectroscopic analysis of the earth atmosphere take the sun as an illumination and calibration source and compare the sun observations with the backscattered radiance of the earth atmosphere. In very simple terms it can be stated that the earth radiance is compared to the sun irradiance, thus the "Bi-Directional Scattering Function" BSDF of the earth $\left(B S D F_{\text {earth }}=\right.$ radiance_earth / irradiance_sun $)$ is determined. Figure 1-1 illustrates this procedure:
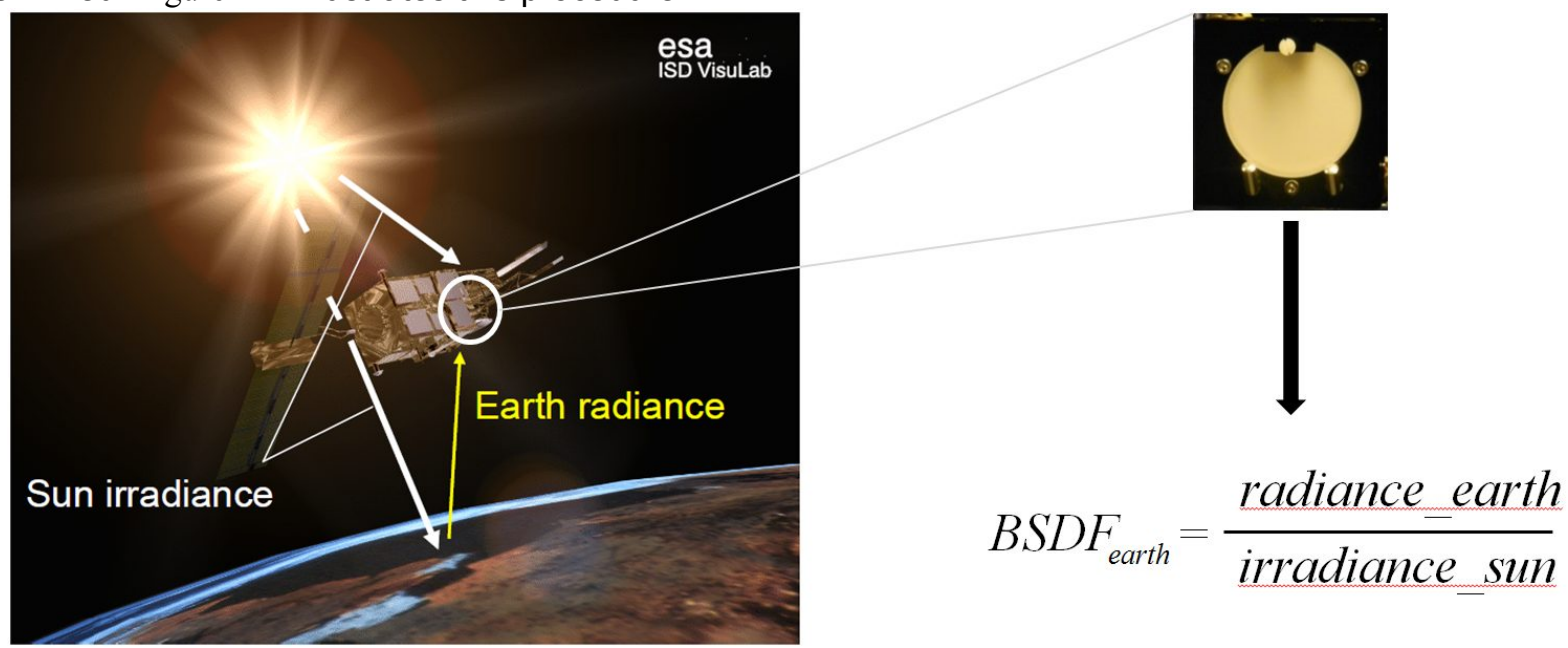

Figure 1-1: Illustration of measuring the sun irradiance and earth radiance by an earth observation instrument (credits image: ESA). The small image on the right shows a solar diffuser (credits: TNO) 
The solar irradiance is needs to be measured along the same optical path. This is realized by using a socalled on-board "solar diffuser", a core element in the calibration unit. This diffuser, illuminated by the sun irradiance, mimics the scattering of the earth and ensures a homogeneous illumination of the spectrometer entrance slit. Its performance is expressed through its scattering properties, the aforementioned "BSDF" (or BTDF in transmission mode or $B R D F$ in reflection mode) and depends on several parameters, such as surface properties, angle of incidence and detection and further. Thus an adequate definition in these terms of variables and parameters is of significant importance. An accurate characterization of the diffuser with regards to its scattering properties is a crucial step in the chain of procedures to ensure high quality remote sensing data products.

The present paper describes TNO's "Absolute Radiometric Calibration Facility ARCF 2.0", in which the aforementioned characterizations are performed. Besides the introduction of the facility, accompanying challenges in order to achieve accurate measurements are discussed and an exemplary case study presented.

\section{ABSOLUTE RADIOMETRIC CALIBRATION FACILITY ARCF 2.0 - SETUP}

The ARCF 2.0 is a test facility located in a dedicated class 100 (ISO 5) clean room that is capable of accurate measurements of the BSDF of diffusers, in particular of flight components where stringent environmental conditions need to be kept. The facility has been used in the past for analyzing components and during calibration campaigns of several earth observation instruments such as the Medium Resolution Imaging Spectrometer (MERIS), SCHIAMACHY, the Ozone Monitoring Instrument (OMI) and GOME-2. Figure 2-1 shows a schematic description of the ARCF measurement set-up. The left image displays the "irradiance" configuration, where the input beam is measured directly, while the right image shows the "radiance" configuration, where the scattering properties of a diffuser are recorded.
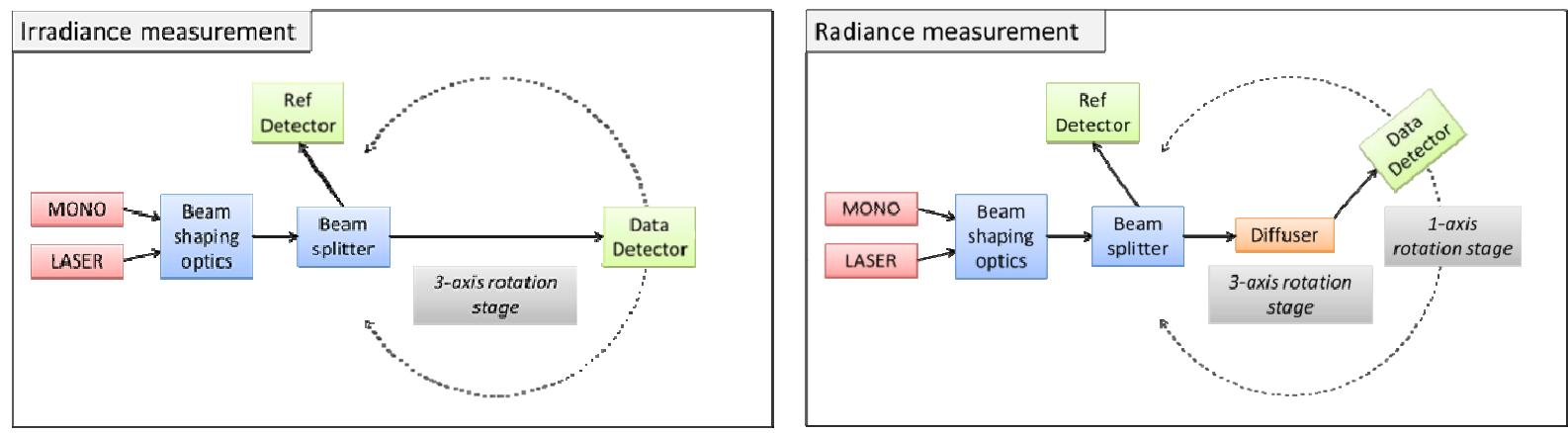

Figure 2-1 Schematic description of the ARCF setup

Two light sources are available, a monochromator and a tunable laser system. The in-house developed monochromator generates a wavelength tunable light beam by a Xe lamp. The beam enters a computer controlled double prism monochromator that is capable to transmit spectral bands in the range from 240 to $2400 \mathrm{~nm}$. The light exits then the monochromator as a uniform collimated beam with a maximum diameter of $40 \mathrm{~mm}$.

The laser system is a tunable OPO system with a wavelength range from $210 \mathrm{~nm}$ to $2600 \mathrm{~nm}$, a pulse length of $10 \mathrm{~ns}$ and a repetition rate of $1 \mathrm{kHz}$.

In both cases the transmitted light is directed towards the optical rail with the data detector and the sample under test. A reference detector continuously monitors the irradiance via a beam-splitter. Both the reference and data detector assemblies contain the same components including telescope, detectors from the same manufacturing batch, and synchronous readout.

Two different detector types assure measurements in the full mentioned wavelength regime: A silicon detector for the UV/VIS/NIR domain between $200 \mathrm{~nm}$ and $1100 \mathrm{~nm}$ and an Indium-Gallium- Arsenide detector for the SWIR range up to $2400 \mathrm{~nm}$. Furthermore the possibility is given to measure with un-polarized and polarized light, for source and for detection.

The data detector assembly is decoupled from the sample motion system and is positioned on an arm capable of a $360^{\circ}$ rotation in a horizontal plane around the diffuser under test. The sample is mounted in a cradle that allows rotation around 3 axes. Hence the entire sample and detector motion system offers 4 degrees of freedom, allowing "out-of-plane" 
measurements. This important feature ensures a characterization of the diffuser component under representative illumination conditions in orbit.

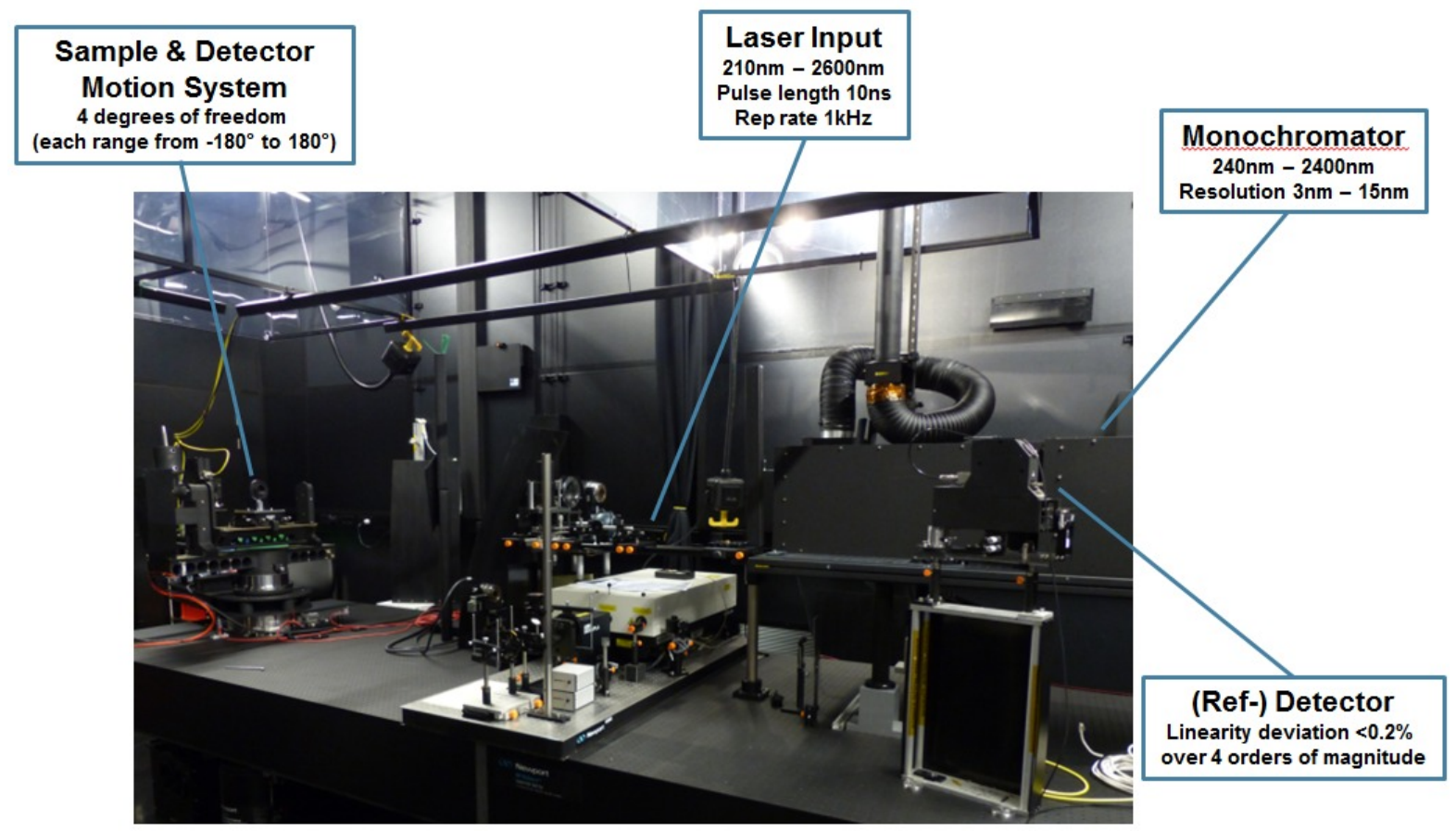

Figure 2-2 Image of the ARCF 2.0 setup with characteristics of key components

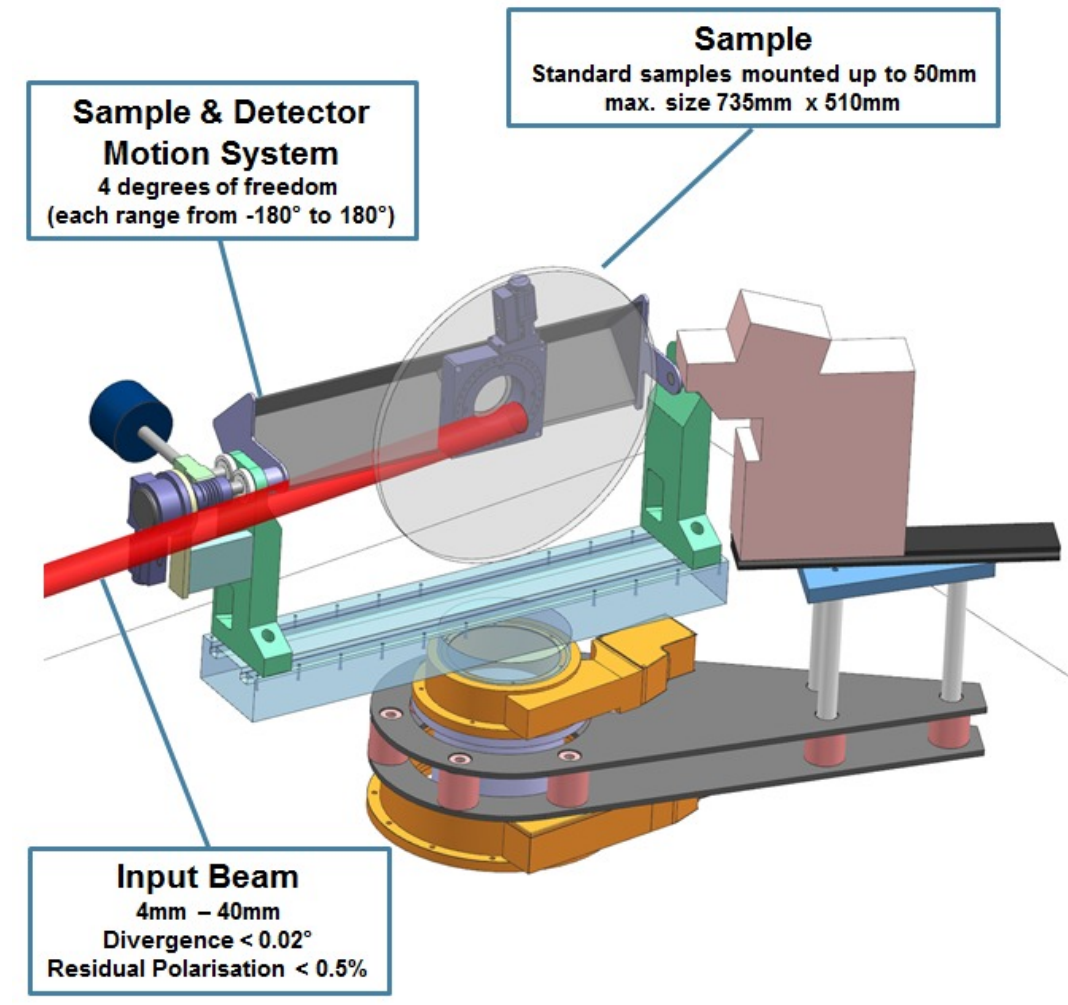

Figure 2-3 Sample and detector motion system of the ARCF 2.0 


\subsection{UNCERTAINTY CONTRIBUTIONS}

To achieve the best possible BSDF measurement, it is essential to fully know the setup, identify all its error contributions and apply according corrective procedures if necessary. TNO invests a large amount of time and effort to identify these contributions and thus to ensure accurate measurements. A detailed description of each potential error source, its determination and correction would be out of scope of this manuscript. For details the reader is encouraged to contact the author. The following table lists some of the setup related error contributions.

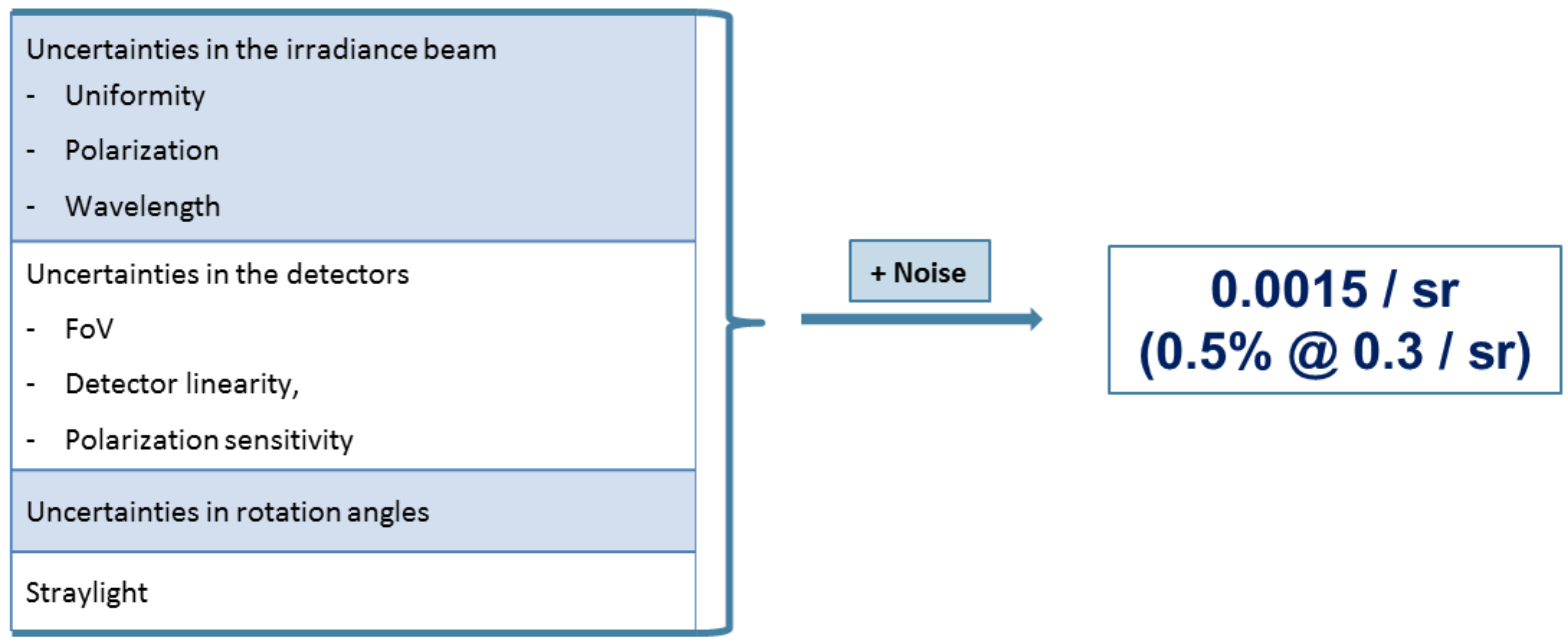

Figure 2.1-1 List of potential error contributions

The characterization of the setup itself occurs in regular intervals, but some high accuracy measurements require additional characterization measurements. As mentioned above the table lists setup related error sources. For the overall error budget the noise needs to be considered as well. Overall the system has the capability of acquiring data with an accuracy of $0.0015 / \mathrm{sr}$. For example, for a spectralon based component with a potential BSDF of $0.3 / \mathrm{sr}$ this value relates to an error of $0.5 \%(1 \sigma)$.

\subsubsection{UNCERTAINTY CONTRIBUTIONS - EXAMPLE}

The criticality of the accurate identification of potential error sources is described in an example, in this case with regards to the beam homogeneity determination.

The aperture of the (in-house designed) detector is "overfilled", i.e. the emitting diffuser spot size is larger than the aperture of the detector. This is partly based on the history of TNO being involved with space applications. For these applications a full representative test of a flight component is desired, including boundary effects of the diffuser mount, meaning that the same illumination conditions should be used as to be expected in orbit. Furthermore, the detector design is optimized for a small $\mathrm{FoV}$ of $1.2^{\circ}$. The overfilled approach makes the setup less sensitive to environmental straylight. This is most important for a so-called "Quasi Volume Diffusers (QVD's)". TNO has a heritage on the design, manufacturing and characterization of these particular components for calibration units of earth observation instruments. A QVD is basically a (mostly) fused silica component with 2 or more roughened surfaces and possibly a coating, if used as a reflective QVD (see fFigure 2.1.1-1). Due to its multiple surfaces and strong scattering properties it becomes more preferable to have an overfilled configuration. 

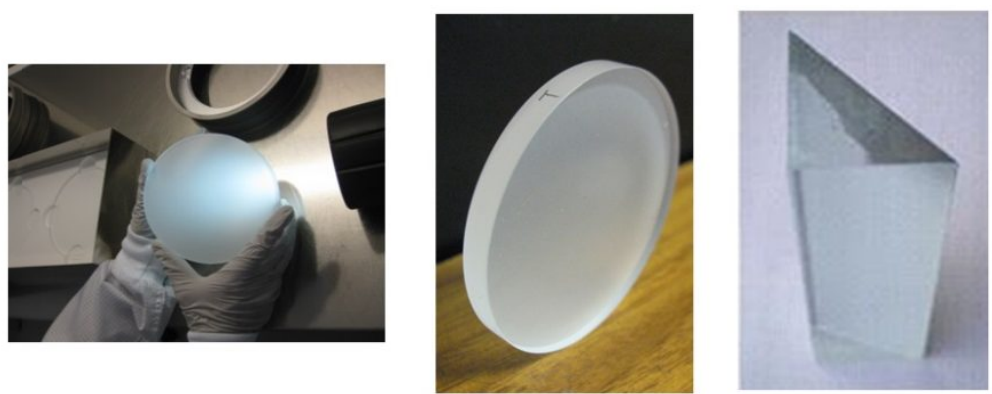

Figure 2.1.1-1 Examples of QVD's (credit TNO)

The choice of an overfilled aperture configuration however has further consequences, such as requiring a good knowledge of the beam homogeneity. This necessity is discussed exemplary on the following effect, called "beam homogeneity translation". For an initial basic explanation please refer to the following figure.

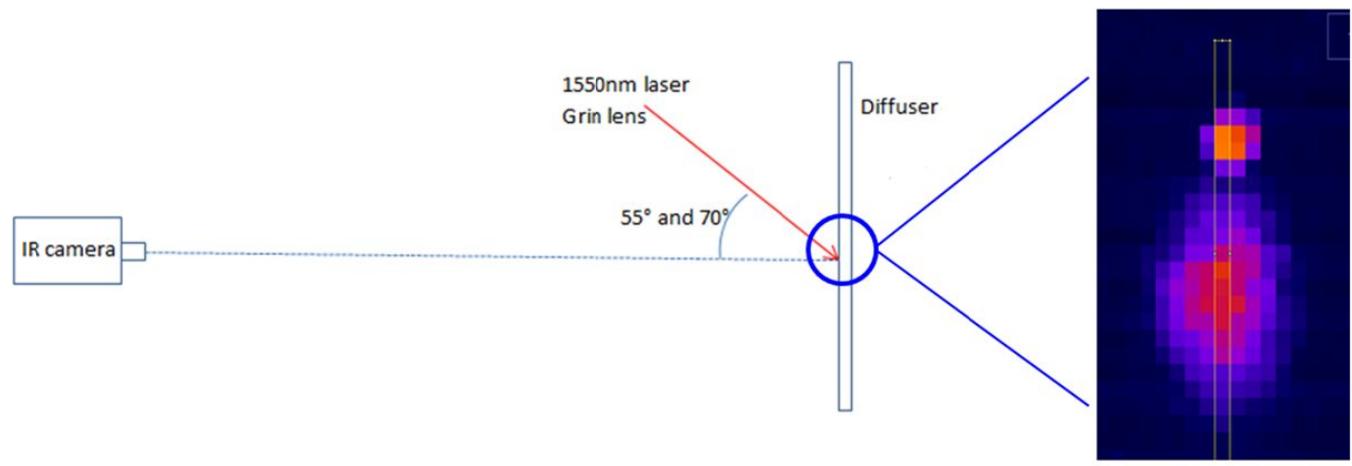

Figure 2.1.1-2 QVD is illuminated with a laser spot, the IR camera observes 2 spots (front plane and back surface)

A reflective QVD, made of fused silica with an aluminum coating is illuminated by a laser (in the present case @ $1550 \mathrm{~nm}$ ), the illumination area is then observed with an IR camera. As can be seen in the right image, the camera observes 2 spots, one from the front plane, one (shifted and more scattered) from the back surface. As we recall the BSDF (in its most basic terms) is the ratio between the radiance and irradiance (BSDF = radiance / irradiance). The following figure indicates how a shifted radiance induces an incorrect calculation of the BSDF, if an inhomogeneous beam is applied. The described beam shift depends on different parameters, such as the thickness of the diffuser and the angle of incidence. In order to determine and correct this shift, a model is applied considering refracting and scattering effects, when the beam penetrates the diffuser.

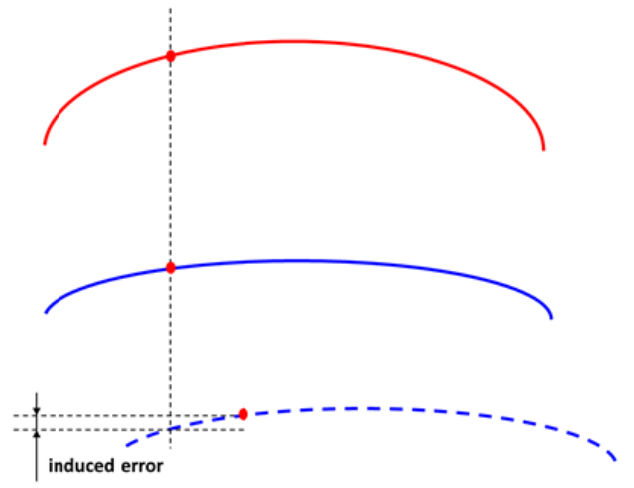

IRR

RAD

RAD_shifted

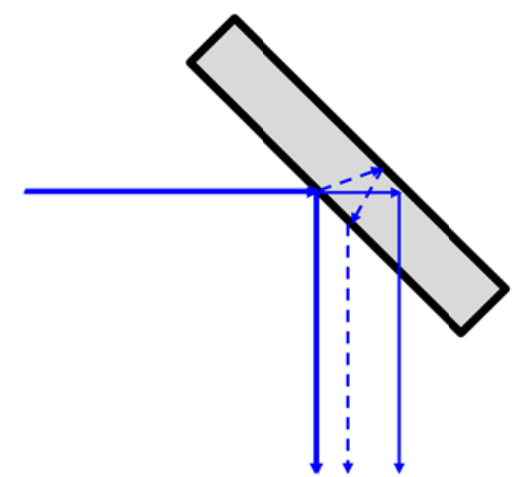

Figure 2.1.1-3 Beam homogeneity translation on a reflective QVD 
Thus, despite of the advantages an overfilled aperture provides, it is required to have a very good knowledge of the beam profile. Characterizing the beam homogeneity is therefore a standard procedure before any diffuser measurement (Figure 2.1.1-4).

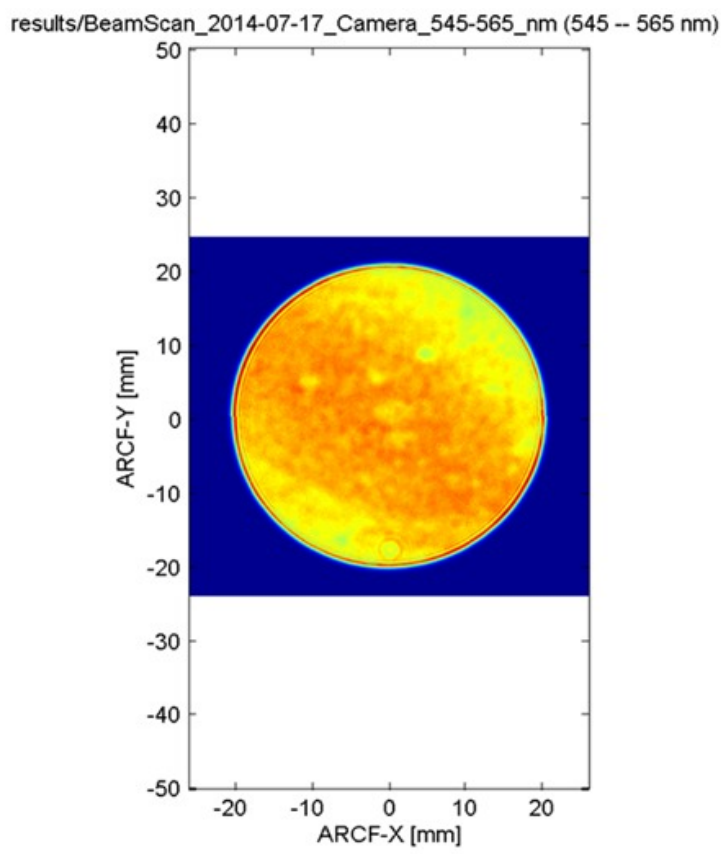

Figure 2.1.1-4 Beam profile measurement within ARCF 2.0 characterization procedures

\section{MEASUREMENTS WITH QVD}

As mentioned above the main application of the ARCF is the accurate characterization of diffusers. However, it is worth to mention that TNO is not only involved in the characterization of diffusers, but also in the design of such components. As mentioned before, in the recent years TNO has tended increasingly towards QVD's. The reason is twofold: First, less degradation in orbit compared to other diffuser types such as spectralon or aluminum diffusers, second, smaller nonuniformities in the recorded spectra in terms of spectral features.

One drawback of QVD's however is the still existing lack of knowledge of the interaction between diffuser properties and performance. The performance of a QVD depends on several parameters, such as angle of incidence and detection, wavelength range, material, surface properties and further.

Out of the variety of these conditions, a design of a component is needed that is most suitable for the individual mission. The image below illustrates this matter.

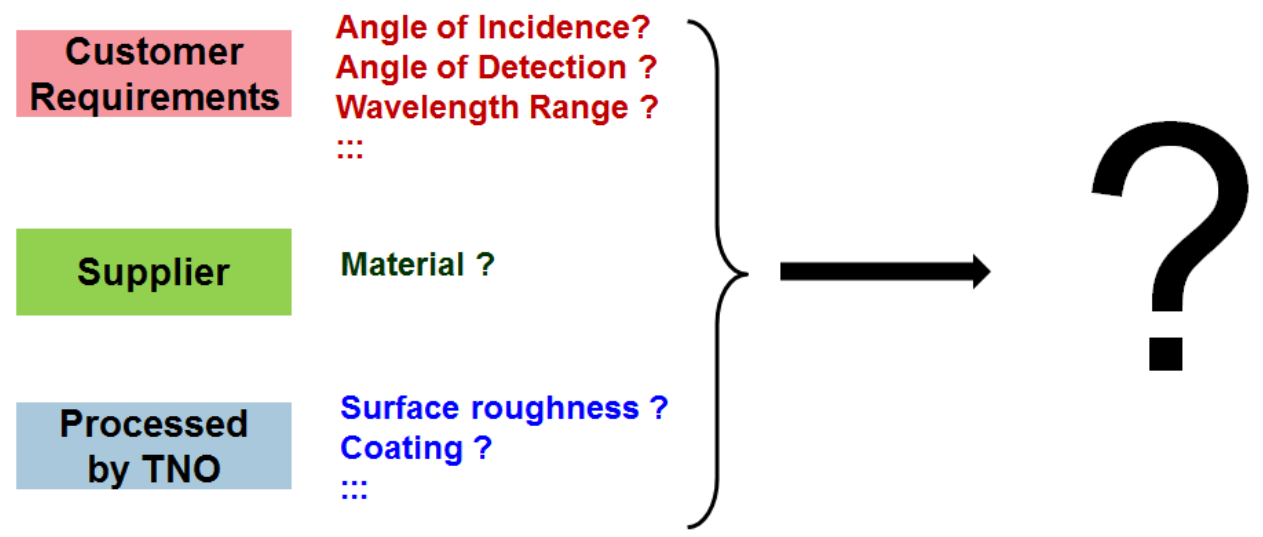

Figure 3-1 Combination of parameters for required diffuser performance 
In the recent past the ARCF has thus been a valuable research tool in the investigation of diffuser properties and according scattering behavior. For example, the following graphics shows various BSDF measurements of diffusers with varying surface roughness [1].
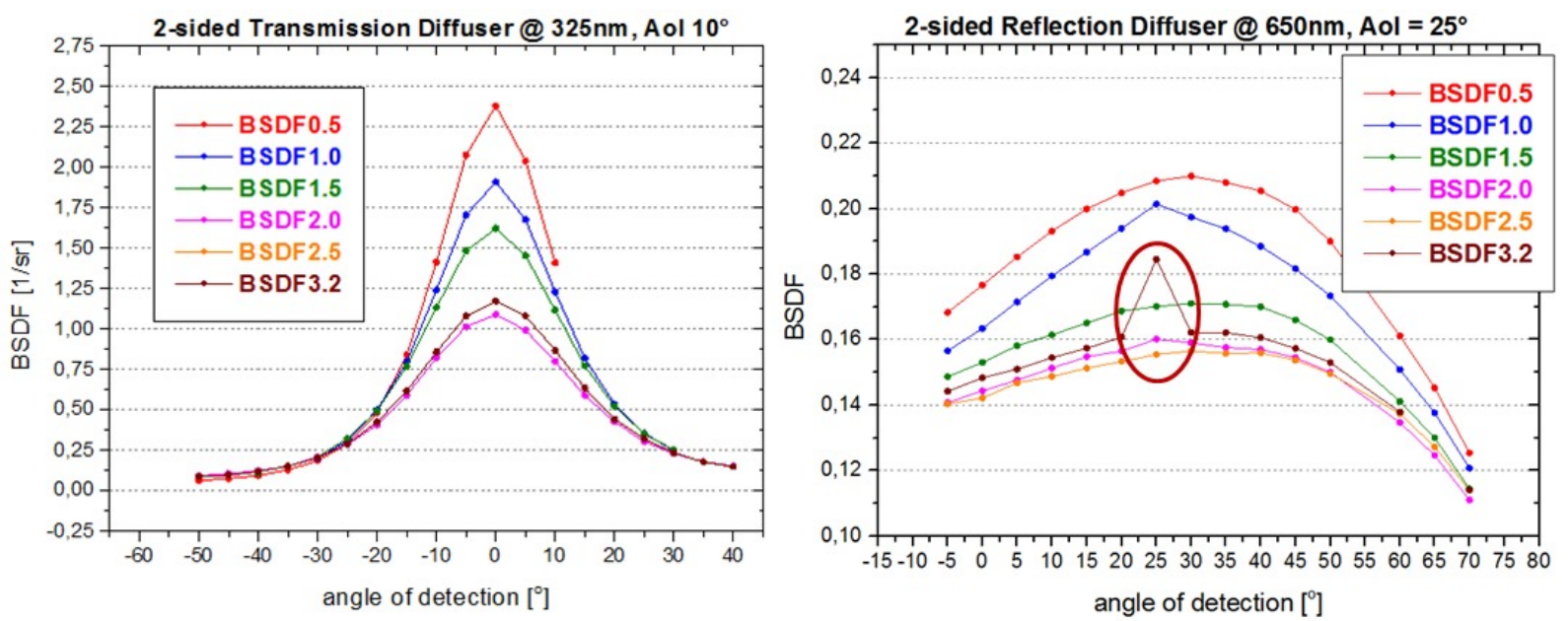

Figure 3-2 BSDF of a transmission and reflection QVD in dependence of surface roughness

Please note that this image is exemplary. For a detailed discussion please refer to [1]. The numbers in the graphics relate to the surface roughness in terms of $R_{q}$ in $\mu \mathrm{m}$. In the right image it can be seen, how a surface defect or incomplete grinding process can be detected through a BSDF analysis.

\section{REFERENCES}

[1] Gür, B., van Brug, H., Xu, M., Vela, E., "Diffusers, properties, and performance in BRDF”, Proc. SPIE 920510 (2014) 